\section{Linfoma anaplástico de células grandes CD30 positivo cutáneo primario: reporte de un caso}

\author{
CRISTIÁN NAVARRETE-DECHENT ${ }^{1}$, MONTSERRAT MOLGÓ ${ }^{1}$, \\ MAURICIO SANDOVAL-OSSES ${ }^{1}$, SERGIO GONZÁLEZ ${ }^{2}$
}

\section{Primary cutaneous anaplastic large cell lymphoma. Report of one case}

Primary cutaneous anaplastic large cell lymphoma (PCALCL) is within the spectrum of cutaneous CD30-positive lymphoproliferative disorders. It presents as localized or multifocal tumors or plaques and carries an excellent long-term prognosis even in cases with regional and/or ipsilateral lymph node involvement or in cases of recurrent disease. We report a 34 year-old female with a thigh lesion. Skin biopsy confirmed the diagnosis of PCALCL. The patient was strictly monitored but no treatment was instituted and the tumor regressed spontaneously. After 24 months of follow-up the patient remains free of disease without new lesions.

(Rev Med Chile 2015; 143: 1351-1355)

Key words: Lymphoma; Primary Cutaneous Anaplastic Large Cell Lymphoma; Prognosis; Skin; Treatment.
'Departamento de Dermatología, Escuela de Medicina, Pontificia Universidad Católica de Chile, Santiago, Chile.

2Departamento de Anatomía Patológica, Escuela de Medicina, Pontificia Universidad Católica de Chile, Santiago, Chile.

Conflictos de interés: Ninguno

Recibido el 16 de julio de 2015 aceptado el 9 de septiembre de 2015.

\section{Correspondencia a:} Cristián Navarrete-Dechent, MD. Departamento de Dermatología, Centro Médico San Joaquín, Escuela de Medicina, Pontificia Universidad Católica de Chile.

Dirección: Av. Vicuña Mackenna 4686. Macul, Santiago, Chile. Teléfono: (56 2) 3548659 Fax: (56 2) 5529974 ctnavarr@gmail.com
L os trastornos linfoproliferativos CD30+ primarios cutáneos (TLPCD30+) son la segunda forma más frecuente de linfomas cutáneos de células T (LCCT). Corresponden a un espectro de enfermedades que incluyen la papulosis linfomatoide y el linfoma anaplástico de células grandes primario cutáneo (LACGPC) ${ }^{1,2}$. El linfoma anaplástico de células grandes (LACG) ocurre como dos entidades clínicamente distintas: el LACGPC y el LACG nodal/sistémico.

El LACGPC se manifiesta como tumores o placas ulceradas, de crecimiento rápido, en forma solitaria o agrupada y posee un excelente pronóstico $^{1,2}$. Su contraparte nodal/sistémica (LACG nodal) es un linfoma agresivo que requiere tratamiento con poliquimioterapia. ${ }^{1}$

\section{Caso clínico}

Mujer de 34 años, sin antecedentes mórbidos; consultó por lesión asintomática en muslo izquierdo de 1 mes de evolución. Se trató como piodermia con múltiples antibióticos orales, sin respuesta. Al examen, presentaba un tumor eritematoso de $5 \times 8$ $\mathrm{cm}$, con pequeñas ulceraciones y erosiones (Figura 1). A la compresión no hubo salida de exudado ni de granos. Además, presentaba dos adenopatías inguinales ipsilaterales, no dolorosas, de aproximadamente $1 \mathrm{~cm}$. No se palparon otras adenopatías ni visceromegalia. Se planteó el diagnóstico de un linfoma cutáneo y de un actinomicetoma, por lo que se solicitaron exámenes serológicos, una tomografía computarizada (TAC) con contraste de tórax, abdomen y pelvis (TAC-TAP), múltiples cultivos bacterianos-micológicos y una biopsia de piel. Tenía hemograma, deshidrogensa láctica (LDH) y calcio sérico dentro de rangos normales; además de serología para HTLV-1 negativa. El TAC-TAP mostró sólo las adenopatías ipsilaterales pesquisadas clínicamente.

La histopatología mostró un tumor dérmico ulcerado, compuesto por células linfoides grandes, con pleomorfismo nuclear y mitosis atípicas (Figura 2). Además, había abundantes eosinófilos. 


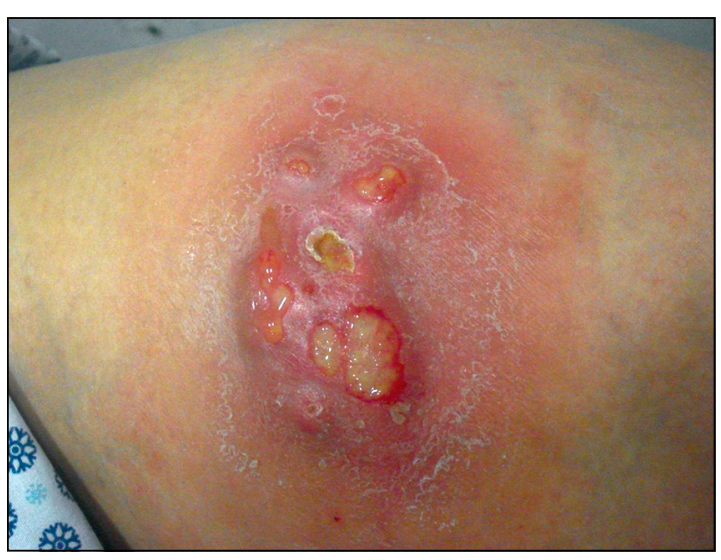

Figura 1. Tumor eritematoso, ulcerado, cubierto de fibrina en algunas zonas, sobre muslo izquierdo.
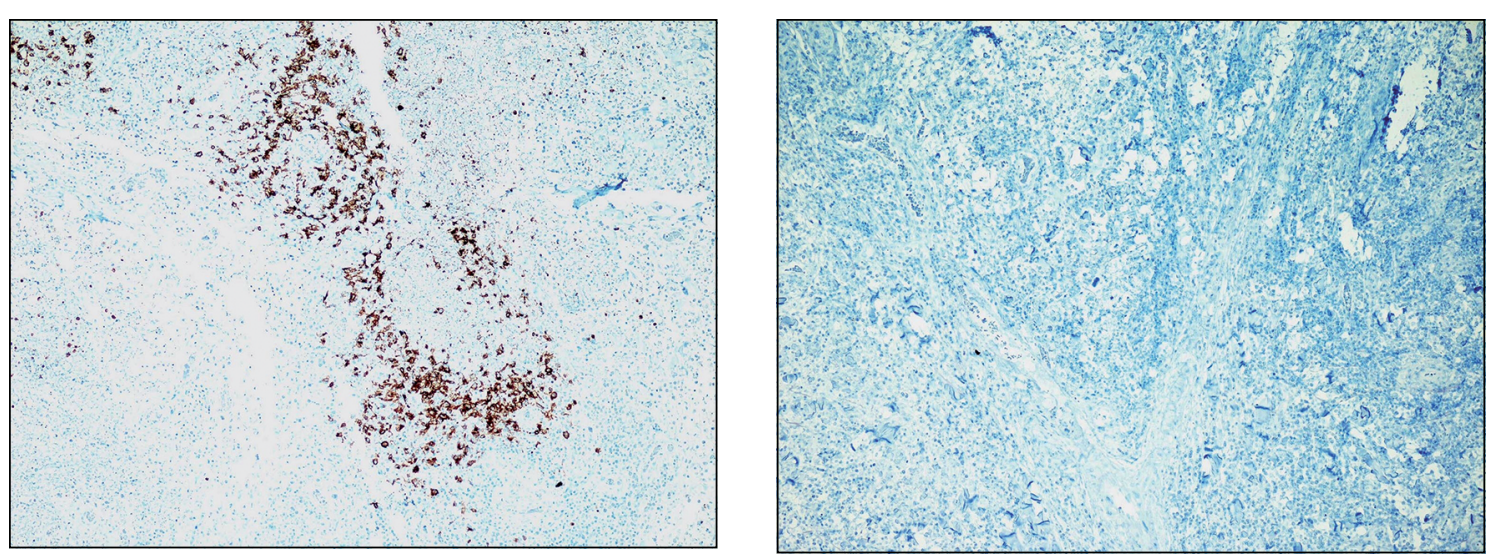

Figura 3. A: Inmunohistoquímica que muestra positividad para CD30 en más de 75\% de las células grandes; B: Inmunohistoquímica que muestra negatividad para ALK-1 en las células grandes.

El estudio inmunohistoquímico (IHQ) mostró reacción positiva para $\mathrm{CD} 30$ en las células grandes (Figura $3 \mathrm{~A}$ ) y reacción negativa para ALK-1 (Figura 3B) y CD25. Se solicitó además una biopsia de médula ósea, que mostró arquitectura y celularidad normales.

Con todos estos elementos se diagnosticó un LACGPC. Se planteó realizar radioterapia (RT) local, pero cuando la paciente volvió a consultar al mes, presentaba clara disminución de tamaño de la lesión del muslo, por lo que no se inició tratamiento. Se controló cada 4 meses y a los 24 meses de seguimiento se encontraba en regresión total (Figura 4).

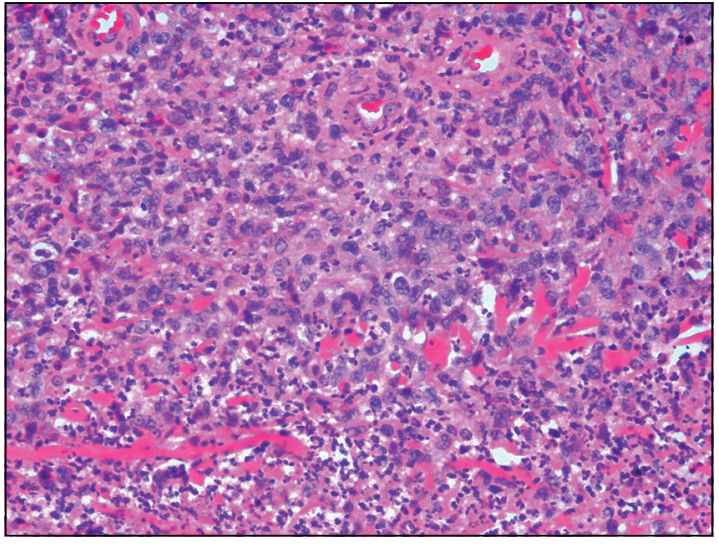

Figura 2. Biopsia de piel que muestra células grandes, con pleomorfismo nuclear, núcleos de diferentes tamaños y formas; todo esto sugerente de un infiltrado linfoide atípico (fijadas en formalina, incluidas en parafina, tinción H\&E, 40X).

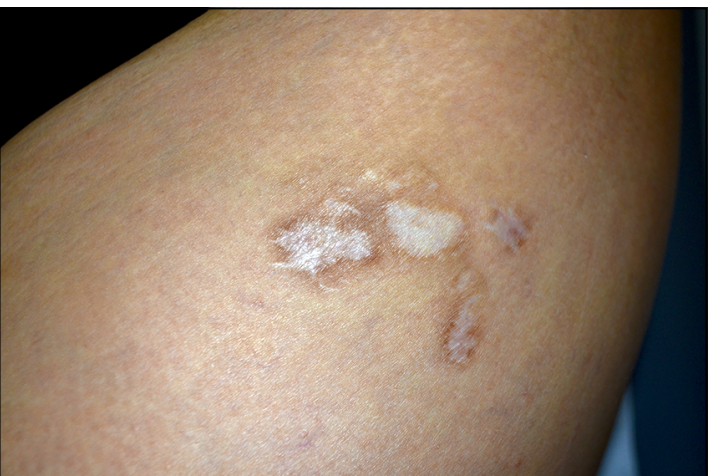

Figura 4. Cicatriz residual en zona del muslo de la paciente 1 año posterior a la lesión inicial. No se palpaban adenopatías. 


\section{Discusión}

Los LCCT son trastornos linfoproliferativos que se caracterizan por la acumulación clonal de linfocitos T neoplásicos en la piel ${ }^{3}$, considerándose "cutáneo primario" a aquel que está limitado a la piel al momento del diagnóstico. ${ }^{4}$ Los TLPCD30+ representan $30 \%$ de todos los LCCT, del mismo modo, el LACGPC corresponde a 1,7\% de todos los linfomas no-Hodgkin (LNH) periféricos y a $8 \%$ de todos los $\mathrm{LCCT}^{5}$. Su incidencia es mayor en la sexta década de vida con una mediana de 61 años (rango 44-87 años) $)^{2,4}$, con leve predominio masculino $(2-3: 1)^{4,5}$. La presentación multifocal (más de 2 sitios anatómicos diferentes o a más de $15 \mathrm{~cm}$ de distancia) se describe en 14-20\% de los casos ${ }^{2,4}$.

Las lesiones se ubican frecuentemente en cabeza, cuello, mitad superior del tronco y extremidades superiores ${ }^{2,4}$. En nuestra paciente la lesión inicial estaba en el muslo: algunos autores afirman que esta ubicación sería un factor de mal pronóstico ${ }^{6,7}$.

El diagnóstico se realiza mediante biopsia de piel; ésta debe incluir hipodermis ${ }^{1}$. El análisis histológico mostrará infiltrado linfocítico dérmico con células grandes anaplásticas, núcleo redondo-oval, irregular, indentado y nucléolo prominente. La IHQ muestra un patrón CD3+, CD4+, CD8-, y CD30+. Por definición, CD30 debe expresarse en al menos $75 \%$ de las células linfoides grandes ${ }^{1}$. El antígeno ALK-1 (anaplastic lymphoma kinase) debe ser negativo. Este es activado frecuentemente debido a la translocación cromosomal no aleatoria $t(2 ; 5)$, causando la fusión del gen de la nucleoplasmina con un receptor de tirosina kinasa, debe ser negativo. Destacamos la importancia del marcador ALK-1, debido a que ayuda a hacer la distinción entre un LACG sistémico ${ }^{8,9}$ con compromiso cutáneo secundario y un LACGCP verdadero. Ambos tipos de linfoma comparten la misma histología con hojas cohesivas de células linfoides grandes que expresan CD30. Sin embargo, clínicamente el LACG es un linfoma agresivo, con compromiso primario de ganglios linfáticos y con compromiso de la piel en $20 \%$ de manera secundaria y en estadios tardíos (además de hueso, médula ósea, hígado y pulmones) ${ }^{2}$. La expresión de ALK-1 en un paciente con diagnóstico de LACGCP debe hacer sospechar que se trata de un LACG y buscar compromiso sistémico, pese a que se han descrito casos de LACGCP con expresión de ALK-1 (Tabla 1) $)^{2,4}$.

La guía de la European Organization for Research and Treatment of Cancer, la International Society fot Cutaneous Lymphomas y de la United States Cutaneous Lymphoma Consortium recomienda estudiar los LACGPC con un hemograma con recuento diferencial, un estudio de química sanguínea que incluya LDH y serología para HTLV- ${ }^{1}$. Queremos resaltar la importancia de solicitar la serología de HTLV-1 debido a que la leucemia-linfoma de células $\mathrm{T}$ del adulto asociada a HTLV-1 puede

Tabla 1. Comparación entre linfoma anaplástico de células grandes primario cutáneo y linfoma anaplástico de células grandes nodal

\begin{tabular}{|c|c|c|}
\hline & $\begin{array}{l}\text { Linfoma anaplástico de células } \\
\text { grandes cutáneo primario CD30+ }\end{array}$ & $\begin{array}{l}\text { Linfoma anaplástico de células } \\
\text { grandes nodal }\end{array}$ \\
\hline Clínica & Pacientes adultos, sexta década de vida & $\begin{array}{l}\text { Si ALK-1 positivo afecta a adultos jóvenes (tercera } \\
\text { década de vida). Sexta década si ALK-1 negativo }\end{array}$ \\
\hline Compromiso extranodal & $\begin{array}{l}\text { Lesiones localizadas en piel, focales o } \\
\text { multifocales } \\
\text { Compromiso extracutáneo raro } \\
(10-13 \%), \text { principalmente ganglios } \\
\text { linfáticos locales }\end{array}$ & $\begin{array}{l}\text { Si ALK-1 positivo compromiso extranodal en } 60 \% \\
\text { pacientes. Piel órgano más frecuentemente } \\
\text { comprometido en } \sim 20 \% \text {; el compromiso ocurre } \\
\text { en etapas avanzadas (III y IV). Otros órganos: } \\
\text { pulmones, médula ósea, hígado y hueso } \\
\text { En casos con ALK-1 negativo compromiso de piel es } \\
\text { principalmente subcutáneo y tardío }\end{array}$ \\
\hline $\begin{array}{l}\text { Tasa de supervivencia a } \\
5 \text { años }\end{array}$ & $\sim 90 \%$ & $\begin{array}{l}\text { Si expresa ALK-1 mejor pronóstico }(70-80 \%) \text { que s } \\
\text { ALK-1 negativo }(40-50 \%)^{8,9}\end{array}$ \\
\hline $\begin{array}{l}\text { Patología e inmuno- } \\
\text { histoquímica }\end{array}$ & Ausencia de expresión de ALK-1 & Expresan ALK-1 la mayoría de las veces \\
\hline
\end{tabular}


simular clínica e histopatológicamente un LACGPC; la primera presenta un tratamiento, curso y pronóstico completamente diferente y ominoso y debe siempre considerarse en el diagnóstico diferencial de cualquier LCCT $^{10,11}$. Se debe solicitar una TAC-TAP con contraste para evaluar compromiso extra-cutáneo, esto debido a que hasta $10-13 \%$ de los pacientes tendrá compromiso sistémico (incluyendo las adenopatías regionales) ${ }^{1,5}$. El valor complementario de la tomografía por emisión de positrones (PET) es discutido ${ }^{1,12}$.

Otro punto controversial es la biopsia de médula ósea; su realización es opcional y debiese reservarse solamente para los casos con anormalidades hematológicas no explicadas, y en aquellos con compromiso extracutáneo en los exámenes radiológicos. Podría realizarse en pacientes con tumores multifocales ${ }^{1}$. En nuestro paciente se realizó el estudio mostrando arquitectura y celularidad normal. En un estudio de 107 pacientes, ninguno presentó compromiso de la médula ósea por el LACGCP ${ }^{13}$.

La etapificación no debe hacerse mediante el sistema de Ann-Arbor, debido a que los tumores multifocales serán categorizados incorrectamente como etapa IV. Deben estratificarse con la etapificación para LCCT diferentes a micosis fungoides y síndrome de Sézary (Tabla 2$)^{14}$.

No existe terapia curativa para este grupo de linfomas; sin embargo, presentan un excelente pronóstico a 5 años con una tasa de supervivencia de $90 \%(76-96 \%)^{1,5}$. El $25-44 \%$ de los pacientes tendrá regresión espontánea de las lesiones, lo cual debe tenerse en consideración al momento de decidir el tratamiento ${ }^{1,2,4}$. La auto-resolución se asocia a un pronóstico favorable ${ }^{1}$. Esta capacidad de auto-resolución es una característica única del LACGCP y no se observa en los casos de linfoma anaplástico nodal; siendo una diferencia fundamental con este último ${ }^{15}$.

Debido a que el LACGPC es una enfermedad indolente, las medidas terapéuticas deben estar enfocadas en estrategias no invasivas ${ }^{15}$. En pacientes con lesiones solitarias el uso de RT (dosis de 3.000-3.600 cGy son suficientes) o extirpación quirúrgica son igualmente efectivas con una respuesta completa de $95 \%$ y tasas de recurrencias limitadas a la piel de $40 \%{ }^{1}$. Si los márgenes quirúrgicos son negativos pareciera razonable evitar la combinación con $\mathrm{RT}^{15}$.

Tabla 2. Etapificación propuesta por ISCL/EORTC para linfomas cutáneos diferentes a la micosis fungoides y al síndrome de Sézary. Adaptada de Kim et al. ${ }^{12}$

\begin{tabular}{|ll|}
\hline T1 & Compromiso cutáneo solitario \\
- T1a & Lesión solitaria de $<5 \mathrm{~cm}$ de diámetro \\
- T1b & Lesión solitaria de $>5 \mathrm{~cm}$ de diámetro \\
T2 & Compromiso cutáneo regional: lesiones múltiples limitadas a 1 segmento corporal o 2 segmentos corporales continuos \\
- T2a & Todas las lesiones confinadas a un área circular $<15 \mathrm{~cm}$ \\
- T2b & Todas las lesiones confinadas a un área circular entre 15 - $30 \mathrm{~cm}$ \\
- T2c & Todas las lesiones confinadas a un área circular $>30 \mathrm{~cm}$ \\
T3 & Compromiso cutáneo generalizado \\
- T3a & Lesiones múltiples que comprometen 2 zonas con contiguas \\
- T3b & Lesiones múltiples que comprometen más de 3 sitios anatómicos \\
N & \\
N0 & Sin compromiso nodal clínico ni patológico \\
N1 & Compromiso de 1 grupo de linfonodos periféricos ${ }^{\dagger}$ que drena zona lesional \\
N2 & Compromiso de 2 o más grupos de linfonodos periféricos o compromiso de nodos ajenos a zona de drenaje \\
N3 & lesional actual o previa \\
M & Compromiso de ganglios linfáticos centrales \\
M0 & Sin evidencia de enfermedad linfoide extracutánea \\
M1 & Presencia de enfermedad linfoide extracutánea
\end{tabular}

†Periféricos: antecubitales, cervicales, supraclaviculares, axilares, inguino-femorales y poplíteos. ${ }^{\dagger \dagger}$ Centrales: mediastínicos, hilios pulmonares, para-aórticos, ilíacos. 
Los pacientes con enfermedad multifocal o compromiso de ganglios linfáticos regionales (zona de drenaje de la lesión) presentan un pronóstico similar a los pacientes con enfermedad localizada y no debe usarse quimioterapia (QT) sólo por este motivo ${ }^{1,2}$. En estos casos, se prefiere el uso de metrotrexato en dosis de 15 a $20 \mathrm{mg}$ semanales pese a que la evidencia es de mala calidad ${ }^{1}$. Se asocia a una tasa de respuesta de $87 \%$ con una duración media de respuesta de 10,6 años en $25 \%$ de los pacientes tras la suspensión del fármaco ${ }^{16}$.

Del mismo modo, las recurrencias limitadas a la piel tampoco confieren un peor pronóstico y no requieren un tratamiento diferente al inicialmente indicado. Es habitual que estos pacientes reciban regímenes combinados de QT de manera innecesaria debido a que son malinterpretados como LNH agresivos ${ }^{1}$.

La QT debe reservarse únicamente para pacientes con adenopatías a distancia y compromiso extracutáneo ${ }^{17}$; su uso en las otras situaciones clínicas se asocia a una alta tasa de recurrencias (62\%), a un tiempo libre de enfermedad promedio de 4 meses y a un alto número de efectos adversos ${ }^{1}$.

\section{Referencias}

1. Kempf W, Pfaltz K, Vermeer MH, Cozzio A, Ortiz-Romero PL, Bagot $\mathrm{M}$, et al. EORTC, ISCL, and USCLC consensus recommendations for the treatment of primary cutaneous CD30-positive lymphoproliferative disorders: lymphomatoid papulosis and primary cutaneous anaplastic large-cell lymphoma. Blood 2011; 118: 4024-35.

2. Diamantidis MD, Myrou AD. Perils and pitfalls regarding differential diagnosis and treatment of primary cutaneous anaplastic large-cell lymphoma. Scientific World Journal 2011; 11: 1048-55.

3. Wollina U. Cutaneous T cell lymphoma: update on treatment. Int J Dermatol 2012; 51: 1019-36.

4. Booken N, Goerdt S, Klemke CD. Clinical spectrum of primary cutaneous CD30-positive anaplastic large cell lymphoma: an analysis of the Mannheim Cutaneous Lymphoma Registry. J Dtsch Dermatol Ges 2012; 10: 331-9.

5. Willemze R, Jaffe ES, Burg G, Cerroni L, Berti E, Swerdlow SH, et al. WHO-EORTC classification for cutaneous lymphomas. Blood 2005; 105: 3768-85.

6. Sugaya M, Fujita H, Izutsu K, Oshima K, Takazawa Y, Ohmatsu $\mathrm{H}$, et al. Primary cutaneous anaplastic large cell lymphoma with leg involvement: a case report and review of 11 cases. J Dermatol 2011; 38: 1009-12.

7. Woo DK, Jones CR, Vanoli-Storz MN, Kohler S, Reddy S, Advani R, et al. Prognostic factors in primary cutaneous anaplastic large cell lymphoma: characterization of clinical subset with worse outcome. Arch Dermatol 2009; 145: 667-74.

8. Lage LA, Cabral TC, Costa ReO, Gonçalves MeC, Levy D, Zerbini MC, et al. Primary nodal peripheral T-cell lymphomas: diagnosis and therapeutic considerations. Rev Bras Hematol Hemoter 2015; 37: 277-84.

9. Stein H, Foss HD, Dürkop H, Marafioti T, Delsol G, Pulford K, et al. CD30(+) anaplastic large cell lymphoma: a review of its histopathologic, genetic, and clinical features. Blood 2000; 96: 3681-95.

10. Bittencourt AL, Barbosa HS, Vieira MD, Farré L. Adult T-cell leukemia/lymphoma (ATL) presenting in the skin: clinical, histological and immunohistochemical features of 52 cases. Acta Oncol 2009; 48: 598-604.

11. Bittencourt AL, de Oliveira MEF. Cutaneous manifestations associated with HTLV-1 infection. Int J Dermatol 2010; 49: 1099-110.

12. Kumar R, Xiu Y, Zhuang HM, Alavi A. 18F-fluorodeoxyglucose-positron emission tomography in evaluation of primary cutaneous lymphoma. Br J Dermatol 2006; 155: 357-63.

13. Benner MF, Willemze R. Bone marrow examination has limited value in the staging of patients with an anaplastic large cell lymphoma first presenting in the skin. Retrospective analysis of 107 patients. Br J Dermatol 2008; 159: 1148-51.

14. Kim YH, Willemze R, Pimpinelli N, Whittaker S, Olsen EA, Ranki A, et al. TNM classification system for primary cutaneous lymphomas other than mycosis fungoides and Sezary syndrome: a proposal of the International Society for Cutaneous Lymphomas (ISCL) and the Cutaneous Lymphoma Task Force of the European Organization of Research and Treatment of Cancer (EORTC). Blood 2007; 110: 479-84.

15. Querfeld C, Khan I, Mahon B, Nelson BP, Rosen ST, Evens AM. Primary cutaneous and systemic anaplastic large cell lymphoma: clinicopathologic aspects and therapeutic options. Oncology (Williston Park) 2010; 24: 574-87.

16. Vonderheid EC, Sajjadian A, Kadin ME. Methotrexate is effective therapy for lymphomatoid papulosis and other primary cutaneous CD30-positive lymphoproliferative disorders. J Am Acad Dermatol 1996; 34: 470-81.

17. Oliveira LS, Nobrega MP, Monteiro MG, Almeida WL. Primary cutaneous anaplastic large-cell lymphoma-case report. An Bras Dermatol 2013; 88: 132-5. 